\title{
Pregiudizi alla diffusione della dialisi peritoneale in Italia
}

\author{
Paolo Gabella
}

\author{
Unità Operativa di Nefrologia, Dialisi e Centro per la Calcolosi Renale \\ Ospedale Maunziano, Torino
}

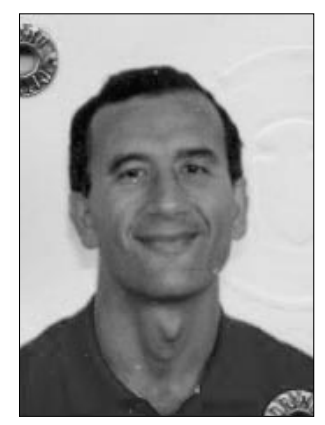

Accanto a
i un'oggettiva
ne f e riori t à
di ulta capacità
ne nel breve pe-
riodo che può
terrorizzare gli
utilizzatori non
esperti; - un'inferiore capacità depurativa nell'unità di tempo, almeno quella che quantizziamo con gli indici depurativi tradizionali, scientificamente validati solo da ricerche svolte in emodialisi, a mio avviso hanno contribuito alla scarsa diffusione della DP sul territorio nazionale una serie di fattori. Cercherò di descrivere i più significativi raggruppandoli in 4 capitoli fondamentali:

\section{Effetto storico}

Problemi organizzativi

Problemi economici e sociali

Problemi ideologici

\section{Effetto storico}

Nascere 15-20 anni dopo una disciplina ad alto contenuto tecnologico, conosciuta da tutti e affermata come terapia che salva la vita è un ritardo che si paga a caro prezzo; per affermarsi bisogna avere dei vantaggi tecnologici che la peritoneale non ha. Poco importa se ci sono dei vantaggi sociali o umani. Se non intervengono grandi distanze geografiche dal Centro dialisi, come in Canada per esempio, la DP non riesce a decollare. Inoltre prima della CAPD la DP in Centro (IPD con bottiglie di vetro) era già stata sperimentata dai membri più anziani delle équipe nefrologiche con risultati scadenti. Il ricordo di questi risultati deludenti, confrontato con lo sviluppo tecnologico che nel frattempo aveva fatto l'emodialisi ha contribuito a frenarne lo sviluppo.

Nascere dopo significa che, per imporsi o conquistare un proprio ruolo, bisogna sapersi riorganizzare e volere investire risorse nel nuovo: un passo che non tutti i gruppi nefrologici hanno voluto o potuto compiere, anche grazie alle scelte dei loro Primari. Alcuni più lungimiranti, dinamici e innovativi hanno sperimentato, investito nelle innovazioni, altri conservatori, se non feudali, hanno soffocato ogni nuova iniziativa.

\section{Problemi organizzativi}

La dialisi peritoneale funziona meglio nell'ottica di un programma di trattamento integrato dell'uremia, come dialisi di prima scelta, per un ingresso precoce e graduale, con dosi incrementali, al trattamento dialitico, conservando il più a lungo possibile, la funzione renale residua, laddove vi è stretta collaborazione con le altre forme di trattamento dell'uremia. Una cultura individualistica, teorica e un po' facilona come quella italiana, non è un terreno ideale per lo sviluppo di una disciplina pratica che richiede l'attenzione a un insieme di piccoli dettagli, e una cura minuziosa del particolare, mantenendo tuttavia una visione d'insieme delle cose.

La DP richiede inoltre un "nursing" avanzato, applicato con costanza e integrato con le altre professionalità (dietista, psicologo, assistente sociale): ancora una volta il lavoro di gruppo non è certo una peculiarità degli Italiani, subissati da una pletora di medici in competizione che svolgono spesso mansioni che sarebbero meglio espletate da altre professionalità (leggi segretaria, infermiere, dietista, psicologo).

\section{Problemi economici}

L'attenzione ai costi e al buon uso della cosa pubblica non è una caratteristica degli Italiani che continuano a manifestare uno scarso senso civico e un disprezzo delle 
cose di tutti. Importa quindi poco che una dialisi sia più economica dal punto di vista dei materiali, e più favorevole nel rapporto personale-numero di assistiti permettendo un risparmio del personale (che incide fino al $60-70 \%$ del costo del trattamento). Dobbiamo attendere almeno 10 anni dalla nascita della CAPD per analisi comparative serie sui costi, che vengono svolte da quei gruppi dove la dialisi peritoneale è più sviluppata. Ancora oggi la questione rimborso del trattamento dialitico discrimina di fatto i Centri che utilizzano metodiche a basso costo. Anche nello stabilire la dotazione e gli organici per la dialisi a domicilio, se 2-3 infermieri della dialisi peritoneale riescono a gestire una cinquantina di pazienti, mentre in emodialisi ne sono necessari almeno il triplo, difficilmente si presta opportuna attenzione alla necessità di seguire ed educare i pazienti a casa, correndo il rischio di strutturare la dialisi peritoneale con locali, personale e medici di serie B.

\section{Problemi ideologici}

Infine la tirannide, intesa come assenza di scelte o egemonia di una sola disciplina, è una strada molto più facilmente percorribile della democrazia. Educare il paziente e informarlo dei vantaggi e degli svantaggi delle diverse opzioni terapeutiche è un processo lungo e difficile: implica una relazione stabile e duratura fra medico e paziente e tutto sommato profonda, che consuma molte energie. Eppure in Italia la rete degli ambulatori nefrologici è molto estesa, e il tempo per preparare il paziente a questo importante "punto di non ritorno" della sua vita ci sarebbe.

Penso che sia un investimento gratificato da ampi ritorni nella successiva vita dialitica, in quanto la conoscenza profonda della malattia cronica e delle sue problematiche, la partecipazione al trattamento migliora sia la sopravviven- za del paziente che la qualità del rapporto paziente-équipe. Inutile sottolineare come gli studi più recenti dimostrino che la percentuale di pazienti in trattamento a domicilio è inversamente proporzionale al numero di pazienti giunti improvvisamente alla dialisi senza informazione, senza accesso vascolare o peritoneale e costretti a una rapida scelta d'ufficio verso l'emodialisi.

gabellap@hotmail.com 